\title{
ON FREQUENCY RESPONSE OF A HYDRAULIC SERVOMOTOR
}

\author{
By KiYozo SAto
}

\section{§1. Introduction.}

The mechanism of a pilot valve controlled hydraulic servomotor can be explained with reference to Fig. 1 . The flow of oil induced by a displacement of the pilot valve A causes a similar displacement of the piston $B$. It is important in the design of an apparatus like this to investigate how faithfully B follows the displacement of A.

One of the methods often used is to investigate the frequency response of an apparatus; i.e. to investigate the motion of $B$ when A is displaced sinusoidally.

Let $y$ denote the displacement of $\mathrm{B}$ when the displacement of $\mathrm{A}$ is

$x=X \sin \omega t, X, \omega$ : positive constants, $t$ : time.

Then it is known that $y$ satisfies following differential equation:

$$
\begin{array}{ll}
m \frac{d^{2} y}{d t^{2}}+\left(\frac{2 A^{3}}{k^{2} X^{2} \sin ^{2} \omega t}+R A^{3}\right)\left(\frac{d y}{d t}\right)^{2} & \begin{array}{l}
\text { Fig. 1. The mechanis } \\
\text { valve controlled hydr } \\
\text { motor }
\end{array} \\
-\left(A P_{s}-F\right)=0, \quad \text { for } \sin \omega t>0, & \text { for } \sin \omega t<0,
\end{array}
$$

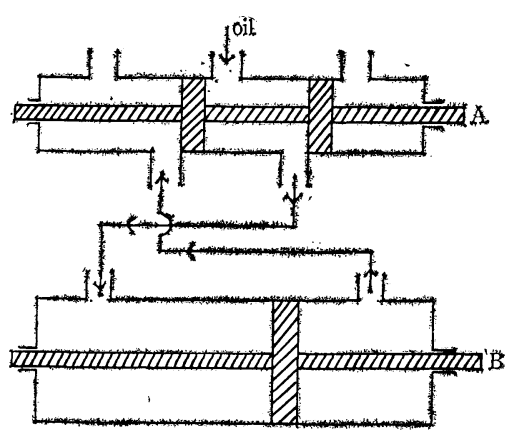

Fig. 1. The mechanism of a pilot valve controlled hydraulic servo-

where $m, A, k, R, P_{s}$ and $F$ are physical constants determined by the characteristics of the apparatus; cf. [1]. Further explanation of the constants will be omitted.

Put

$$
\omega t=\theta, \quad \frac{d y}{d \theta}=u, \quad \frac{2 A^{3}}{m k^{2} X^{2}}=a, \quad \frac{R A^{3}}{m}=b, \quad \frac{A P_{s}-F}{m \omega^{2}}=c .
$$

Then the above differential equation will be reduced to

$$
\frac{d u}{d \theta}+\left(\frac{a}{\sin ^{2} \theta}+b\right) u^{2}-c=0, \quad \text { for } \sin \theta>0,
$$

Received July 13, 1964. 


$$
\frac{d u}{d \theta}-\left(\frac{a}{\sin ^{2} \theta}+b\right) u^{2}+c=0, \quad \text { for } \sin \theta<0
$$

It is known from physical conditions that

$$
0<b<a \ll c \text {. }
$$

The purpose of this paper is to find a solution of (1.1) and (1.2) which represents the motion of the piston $B$.

\section{$\S 2$. The symmetry characters of solutions.}

If $u=f(\theta)$ is one of the solutions of (1.1) and if $g(\theta) \equiv f(\theta-\pi)$, then

$$
\frac{d f(\theta-\pi)}{d(\theta-\pi)}+\left(\frac{a}{\sin ^{2}(\theta-\pi)}+b\right)\{f(\theta-\pi)\}^{2}-c=0,
$$

which can be easily reduced to

$$
\frac{d g(\theta)}{d \theta}+\left(\frac{a}{\sin ^{2} \theta}+b\right)\{g(\theta)\}^{2}-c=0,
$$

showing that $u=g(\theta) \equiv f(\theta-\pi)$ is also a solution of (1.1). This means that when one integral curve of (1.1) is obtained, it can be translated $\pi$ units in the $\theta$ direction to obtain another one.

If $h(\theta) \equiv-f(\theta)$, then

$$
\begin{aligned}
& \frac{d h(\theta)}{d \theta}-\left(\frac{a}{\sin ^{2} \theta}+b\right)\{h(\theta)\}^{2}+c \\
= & -\left[\frac{d f(\theta)}{d \theta}+\left(\frac{a}{\sin ^{2} \theta}+b\right)\left\{f(\theta\}^{2}-c\right]=0,\right.
\end{aligned}
$$

showing that $u=h(\theta) \equiv-f(\theta)$ is a solution of (1.2) when $u=f(\theta)$ is a solution of (1. 1).

From the above, the following is established:

Obtain all the solutions of (1.1) for $0 \leqq \theta \leqq \pi$. Translate them in the $\theta$-direction by $\pi$ and change their sign to obtain all the solutions of (1.2) for $\pi \leqq \theta \leqq 2 \pi$. Connect these two sets of integral curves at $\theta=\pi$ and we obtain all the integral curves of the given differential equation for $0 \leqq \theta \leqq 2 \pi$. Successive translations in the $\theta$ direction by $2 \pi$ will provide the complete set of solutions of (1.1) and (1.2) for $0 \leqq \theta<\infty$. Therefore it suffices to know the behavior of integral curves of (1.1) for $0 \leqq \theta \leqq \pi$ to obtain the complete knowledge of the solutions of (1.1) and (1.2) for $0 \leqq \theta<\infty$. 
In constructing the solution curves by the method stated above, there naturally remains some ambiguity since the connection of the integral curves at $\theta=n \pi$ is still left arbitrary. However, physical consideration will show that, to obtain the solution we are seeking for, it is most plausible to choose the possibly smooth connection at $\theta=n \pi$.

Prior to the investigation of the integral curves of

$$
\frac{d u}{d \theta}+\left(\frac{a}{\sin ^{2} \theta}+b\right) u^{2}-c=0
$$

in the interval $0 \leqq \theta \leqq \pi$, the following fact should be noticed. If $u=f(\theta)$ is one of the solutions of (1.1) and $-f(\pi-\theta) \equiv \varphi(\theta)$, then

$$
\begin{aligned}
& \frac{d \varphi(\theta)}{d \theta}+\left(\frac{a}{\sin ^{2} \theta}+b\right)\{\varphi(\theta)\}^{2}-c \\
= & \frac{d f(\pi-\theta)}{d(\pi-\theta)}+\left\{\frac{a}{\sin ^{2}(\pi-\theta)}+b\right\}\{f(\pi-\theta)\}^{2}-c=0,
\end{aligned}
$$

showing that $u=\varphi(\theta) \equiv-f(\pi-\theta)$ is also a solution of (1.1). So if we rotate an integral curve of (1.1) for $0 \leqq \theta \leqq \pi$ by an angle $\pi$ about a point $\theta=\pi / 2, u=0$, another integral curve of the same equation is obtained.

\section{$\S 3$. Behavior of the solutions at $\theta=0$ and $\theta=\pi$.}

As the discussions of $\S 2$ have shown, we should naturally lay an emphasis upon the study of solution curves of (1.1) for $0 \leqq \theta \leqq \pi$.

A transformation

$$
u=c w / \frac{d w}{d \theta}
$$

will reduce (1. 1) to a linear equation

$$
\frac{d^{2} w}{d \theta^{2}}-c\left(\frac{a}{\sin ^{2} \theta}+b\right) w=0
$$

The indicial equation at a regular singular point $\theta=0$ is

$$
\lambda^{2}-\lambda-a c=0
$$

with two roots

$$
\lambda_{1}=\frac{1+\sqrt{1+4 a c}}{2},
$$




$$
\lambda_{2}=1-\lambda_{1}=\frac{1-\sqrt{1+4 a c}}{2} .
$$

According to the condition (1.3), $\lambda_{1}>0$ and $\lambda_{2}<0$. Further we add an assumption that $\lambda_{1}-\lambda_{2}=\sqrt{1+4 a c}$ is not an integer.

In the vicinity of $\theta=0$, the general solution of (3.1) is expressed in a form

$$
w=\alpha \theta^{\lambda_{1}}(1+\cdots)+\beta \theta^{\lambda_{2}}(1+\cdots)
$$

where $\alpha$ and $\beta$ are constants and the unwritten terms inside the brackets are convergent power series of $\theta$ without constant terms. From this follows that

$$
\begin{aligned}
u & =c w / \frac{d w}{d \theta} \\
& =c \cdot \frac{\alpha \theta^{\lambda_{1}}(1+\cdots)+\beta \theta^{\lambda_{2}}(1+\cdots)}{\alpha \lambda_{1} \theta^{\lambda_{1}-1}(1+\cdots)+\beta \lambda_{2} \theta^{\lambda_{3}-1}(1+\cdots)} \\
& =c \cdot \frac{\alpha \theta^{\lambda_{1}}(1+\cdots)+\beta \theta^{1-\lambda_{1}}(1+\cdots)}{\alpha \lambda_{1} \theta^{\lambda_{1}-1}(1+\cdots)+\beta\left(1-\lambda_{1}\right) \theta^{-\lambda_{1}}(1+\cdots)} .
\end{aligned}
$$

To the solutions of (3.1) with $\beta=0$ corresponds a solution of (1.1):

such that

$$
u=c \frac{\alpha \theta^{\lambda_{1}}(1+\cdots)}{\alpha \lambda_{1} \theta^{\lambda_{1}-1}(1+\cdots)}=c \frac{\theta(1+\cdots)}{\lambda_{1}(1+\cdots)}
$$

$$
\lim _{\theta \rightarrow 0} \frac{u}{\theta}=\frac{c}{\hat{\lambda}_{1}}>0
$$

Except this one, the solutions of (1.1) are written in the form

$$
\begin{aligned}
u & =c \frac{k \theta^{\lambda_{1}}(1+\cdots)+\theta^{1-\lambda_{1}}(1+\cdots)}{k \lambda_{1} \theta^{\lambda_{1}-1}(1+\cdots)+\left(1-\lambda_{1}\right) \theta^{-\lambda_{1}}(1+\cdots)} \\
& =c \frac{k \theta^{2 \lambda_{1}}(1+\cdots)+\theta(1+\cdots)}{k \lambda_{1} \theta^{2 \lambda_{1}-1}(1+\cdots)+\left(1-\lambda_{1}\right)(1+\cdots)}
\end{aligned}
$$

where we have put $\alpha / \beta=k$. Since $2 \lambda_{1}-1=\sqrt{1+4 a c}>0$, and $\lambda_{1}>1$, we have

$$
\lim _{\theta \rightarrow 0} \frac{u}{\theta}=\frac{c}{1-\lambda_{1}}<0 \text {. }
$$

It is thus concluded that all the integral curves of (1.1) tend to zero as $\theta \rightarrow 0$, and only one among them is tangent to the line 


$$
u=\frac{c}{\lambda_{1}} \theta \quad\left(\frac{c}{\lambda_{1}}>0\right)
$$

while the others are all tanget to the line

$$
u=\frac{c}{1-\lambda_{1}} \theta \quad\left(\frac{c}{1-\lambda_{1}}<0\right)
$$

The behavior of the integral curves at $\theta=\pi$ can be easily inferred by a remark mentioned at the end of $\S 2$. Therefore $u \rightarrow 0$ as $\theta \rightarrow \pi$ and only one of the integral curves is tangent to the line

$$
u=\frac{c}{\lambda_{1}}(\theta-\pi)
$$

while the others are all tangent to the line

$$
u=\frac{c}{1-\lambda_{1}}(\theta-\pi)
$$

\section{$\S 4$. Fundamental properties of integral curves.}

Putting $u=0$ in (1. 1), we get

$$
\frac{d u}{d \theta}=c>0 \quad \text { for } \quad u=0 .
$$

Hence:

1) The integral curve has a positive inclination when it crosses the $\theta$-axis.

Rewriting (1.1) in a form

$$
0 \leqq\left(\frac{a}{\sin ^{2} \theta}+b\right) u^{2}=c-\frac{d u}{d \theta}
$$

we immediately have:

2) $d u / d \theta$ is bounded above. Therefore it never happens that $u \rightarrow+\infty$ as $\theta$ increases from 0 to $\pi$.

Differentiating both sides of (1.1) with respect to $\theta$, we get

$$
\frac{d^{2} u}{d \theta^{2}}-\frac{2 a \cos \theta}{\sin ^{3} \theta} u^{2}+2\left(\frac{a}{\sin ^{2} \theta}+b\right) u \frac{d u}{d \theta}=0
$$

Put $d u / d \theta=0$ in this and get 


$$
\frac{d^{2} u}{d \theta^{2}}=\frac{2 a \cos \theta}{\sin ^{3} \theta} u^{2} \begin{cases}\geqq 0 & \text { for } \quad 0<\theta \leqq \pi / 2 \\ \leqq 0 & \text { for } \quad \pi / 2 \leqq \theta<\pi\end{cases}
$$

Therefore

3) $u$ does not attain its maximum between $0<\theta<\pi / 2$, and does not attain its minimum between $\pi / 2<\theta<\pi$.

Our further investigation is essentially based on those properties stated above.

\section{$\S 5$. Definition of the domains $S, S_{1}, S_{2}$.}

As was shown in $\S 3, \lim _{\theta \rightarrow 0} u=0$ and $\lim _{\theta \rightarrow \pi} u=0$ for all the solutions of (1.1) and only one of the integral curves is tangent at $\theta=0$ to the straight line $u=\left(c / \lambda_{1}\right) \theta$. This solution will be denoted by $C_{1}: u=f_{1}(\theta)$. Also the one particular solution which is tangent at $\theta=\pi$ to the line $u=\left(c / \lambda_{1}\right)(\theta-\pi)$ is denoted by $C_{2}: u=f_{2}(\theta)$.

Proposition 1. As $\theta$ increases from 0 to $\pi, f_{1}(\theta)$ increases at first, reaches its maxtmum at $\theta=\theta_{0}>\pi / 2$, and decreases monotonically thereafter to have the limiting values

$$
\lim _{0 \rightarrow \pi} f_{1}(\theta)=0 \quad \text { and } \quad \lim _{\theta \rightarrow \pi} f_{1}^{\prime}(\theta)=\frac{c}{1-\lambda_{1}} .
$$

As $\theta$ decreases from $\pi$ to $0, f_{2}(\theta)$ decreases at first, reaches its minimum at $\theta=\pi$ $-\theta_{0}<\pi / 2$ and then increases to have the limiting values

$$
\lim _{\theta \rightarrow 0} f_{2}(\theta)=0 \quad \text { and } \quad \lim _{\theta \rightarrow 0} f_{2}^{\prime}(\theta)=\frac{c}{1-\lambda_{1}} .
$$

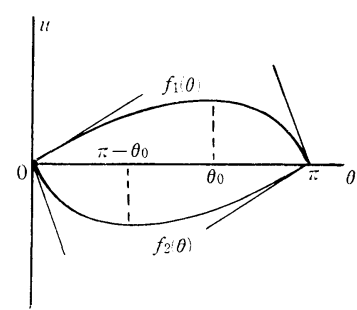

Fig. 2. The curves $C_{1}$ and $C_{2}$

Proof. As $\lim _{\theta \rightarrow 0} f_{1}^{\prime}(\theta)=c / \lambda_{1}>0, f_{1}(\theta)$ increases in the vicinity of $\theta=0$.

As $\theta$ increases from 0 to $\pi, f_{1}(\theta)$ is bounded above as was shown in 2) of $\S 4$ and the curve $C_{1}$ cannot cross the $\theta$-axis with a negative inclination according to 1 ) of $\S 4$. Therefore $0<f_{1}(\theta)<\infty$ for $0<\theta<\pi$. In other words, the curve $C_{1}$ lies in some bounded area of the upper half plane. In addition, $\lim _{\theta \rightarrow 0} f_{1}(\theta)=\lim _{\theta \rightarrow \pi} f_{1}(\theta)$ $=0$. Hence $f_{1}(\theta)$ attains its maxima somewhere between $0<\theta<\pi$. Let the smallest of the $\theta$ 's that make $f_{1}(\theta)$ maximal be $\theta_{0}$. Then $\theta_{0}>\pi / 2$ by 3 ) of $\S 4$. Then, owing also to 3 ) of $\S 4, f_{1}(\theta)$ has no minima for $\theta>\theta_{0}>\pi / 2$. Therefore $f_{1}(\theta)$ is monotonically decreasing for $\theta>\theta_{0}$ and $\lim _{\theta \rightarrow \pi} f_{1}(\theta)=0$. Moreover $f_{1}(\theta)$ being positive for $0<\theta<\pi$, $\lim _{\theta \rightarrow \pi} f_{1}^{\prime}(\theta)$ must be negative. As the value of $d u / d \theta$ at $\theta=\pi$ is either $c / \lambda_{1}>0$ or $c /\left(1-\lambda_{1}\right)<0$, it is proved that 


$$
\lim _{\theta \rightarrow \pi} f_{1}^{\prime}(\theta)=\frac{c}{1-\lambda_{1}}
$$

This proves the truth of our statement concerning the behavior of $f_{1}(\theta)$.

According to the remark stated at the end of $\S 2$, the curve

$$
u=-f_{1}(\pi-\theta)
$$

obtained by rotating the curve $C_{1}$ by an angle $\pi$ about a point $(\pi / 2,0)$ is also one of the integral curves of (1.1) and is tangent at $\theta=\pi$ to the line $u=\left(c / \lambda_{1}\right)(\theta-\pi)$. However, the only integral curve which is tangent at $\theta=\pi$ to the line $u=\left(c / \lambda_{1}\right)(\theta-\pi)$ being $C_{2}$, the statement about the behavior of $C_{2}$ described in Proposition 1 can be immediately derived from above.

We divide the strip

$$
0 \leqq \theta \leqq \pi,-\infty<u<\infty
$$

into three domains-namely the domain enclosed by $C_{1}$ and $C_{2}$, the domain above $C_{1}$ and the domain below $C_{2}$. These domains will be named $S, S_{1}$ and $S_{2}$ respectively.

\section{$\S 6$. Behavior of the integral curves in $S$.}

First the behavior of the integral curves starting from the point $\theta=0, u=0$ into the area $S$ will be investigated. $S$ being surrounded by two integral curves $C_{1}$ and $C_{2}$, these curves cannot go out of $S$ and they stay in $S$ until they reach the point $\theta=\pi, u=0$. Among these solutions, $C_{1}$ is the only curve which is tangent to the straight line $u=\left(c / \lambda_{1}\right) \theta$, and the others are all tangent to the line $u=\left(c /\left(1-\lambda_{1}\right)\right) \theta$. In other words, if one of the latter is denoted by $u=f(\theta)$, it naturally follows that $\lim _{\theta \rightarrow 0} f^{\prime}(\theta)=c /\left(1-\lambda_{1}\right)<0$. So $f(\theta)$ decreases and $f(\theta)<0$ in the vicinity of $\theta=0$.

On the other hand, as $\theta \rightarrow \pi, C_{2}$ is the only integral curve such that $\lim _{\theta \rightarrow \pi} d u / d \theta$ $=c / \lambda_{1}>0$. Therefore for all the other curves $u=f(\theta), \lim _{\theta \rightarrow \pi} f^{\prime}(\theta)=c /\left(1-\lambda_{1}\right)<0$ and thus we are lead to the conclusion that $f(\theta)>0$ in the vicinity of $\theta=\pi$. Therefore as $\theta$ increases from 0 to $\pi, f(\theta)$ decreases at first and then attains a minimum at $\theta=\theta_{1}<\pi$. From 3) of $\S 4$ follows that $\theta_{1}<\pi / 2$. As $f(\theta)>0$ in the vicinity of $\theta=\pi$, $f(\theta)$ must attain its maximum at some point $\theta=\theta_{2}>\theta_{1}$. According to 3) of $\S 4$, $\theta_{2}>\pi / 2$ and $f(\theta)$ cannot have a minimum for $\theta>\theta_{2}>\pi / 2$. So $f(\theta)$ decreases monotonically for $\theta>\theta_{2}$ and tends to the point $(\pi, 0)$. Thus we have reached the following

Proposition 2. Any one of the integral curves starting from the point $(0,0)$ into the domain $S\left(C_{1}\right.$ and $C_{2}$ excluded $)$ is tangent at $(0,0)$ to the curve $C_{2}$, decreases at first, attains its minimum at $\theta=\theta_{1}<\pi / 2$, increases thereafter, attains its maximum at $\theta=\theta_{2}>\pi / 2$ and decreases to reach $(\pi, 0)$ where it is tangent to $C_{1}$. Therefore the

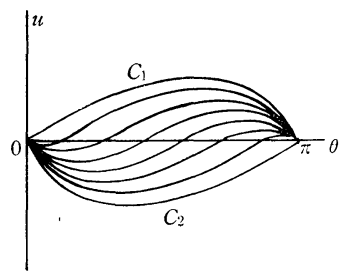

Fig. 3. The integral curves in $S$ domain $S$ is covered with integral curves whose shapes are shown in Fig. 3. 


\section{§7. Behavior of the integral curves in $S_{1}$ and $S_{2}$.}

Next the integral curves starting from $(0,0)$ into the domain $S_{2}$ will be investigated. As they must remain in $S_{2}$, they cannot remain bounded until they reach the line $\theta=\pi$. Because, if so, they must tend to the point $(\pi, 0)$ as $\theta \rightarrow \pi$ where they must naturally be tangent to $C_{2}$. However $C_{2}$ is the only integral curve with this property, such a situation can never arise. Therefore such solutions must tend to $-\infty$ as $\theta \rightarrow \theta_{3}-0$ for some $\theta_{3}<\pi$.

To obtain the integral curves in $S_{1}$, we have only to rotate the integral curves in $S_{2}$ by an angle $\pi$ about a point $(\pi / 2,0)$ owing to the remark at the end of $\S 2$.

\section{$\S 8$. Determination of the desired solution.}

The above investigation clearly indicates the behavior of the totality of the integral curves of (1.1) for $0 \leqq \theta \leqq \pi$. Then, according to the result of $\S 2$, the integral curves of (1.2) for $\pi \leqq \theta \leqq 2 \pi$ can be constructed by translating these curves by $\pi$ units in the $\theta$-direction and changing their sign.

Thus all the integral curves of the given equation for $0 \leqq \theta \leqq 2 \pi$ are obtained. In order to get all the solutions for $0 \leqq \theta<\infty$, it is only necessary to translate them by $2 \pi$ in the $\theta$-direction repeatedly. Integral curves thus obtained are shown in Fig. 4.

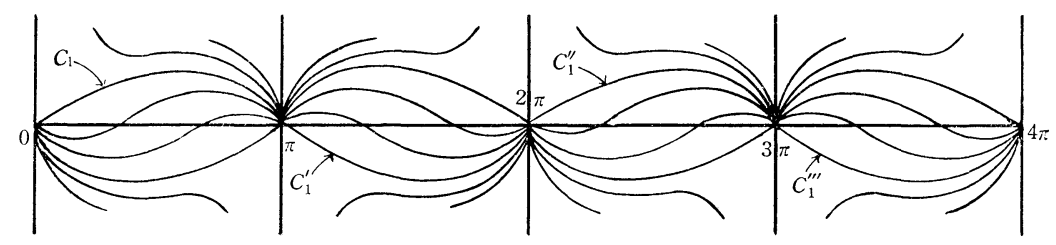

Fig. 4. Totality of the integral curves

In connecting a curve between $(n-1) \pi \leqq \theta \leqq n \pi(n=1,2,3, \cdots)$ with a curve between $n \pi \leqq \theta \leqq(n+1) \pi$, there always occurs discontinuity of respective derivatives at $\theta=n \pi$, whatever integral curves are selected. From a physical point of view, it is reasonable to suppose that actual connection will take place so that the jump of the derivatives at $\theta=n \pi$ is minimized. It may be said that a curve $\mathrm{C}$ obtained by connecting $C_{1}, C_{1}{ }^{\prime}, C_{1}{ }^{\prime \prime}, \ldots$ is physically stable. Here $C_{1}{ }^{\prime}$ is constructed by translating $C_{1}$ in the $\theta$-direction by $\pi$ and changing its sign, $C_{1}^{\prime \prime}$ is constructed by translation of $C_{1}$ in the $\theta$-direction by $2 \pi, C_{1}{ }^{\prime \prime \prime}$ is constructed by translation of $\mathrm{C}_{1}{ }^{\prime}$ in the $\theta$-direction by $2 \pi$ and so on. Whatever solution curve is chosen at $\theta=0$ (excluding the unbounded ones), this solution will finally be connected to $C$ as can be easily seen from Fig. 4.

Therefore, if $u=\varphi(\theta)$ is the equation of the curve $C_{1}$, then the desired solution will be given by 


$$
\begin{array}{ll}
u=\varphi(\theta) & \text { for } \quad 0 \leqq \theta \leqq \pi, \\
u=-\varphi(\theta-\pi) & \text { for } \quad \pi \leqq \theta \leqq 2 \pi, \\
u=\varphi(\theta-2 \pi) & \text { for } 2 \pi \leqq \theta \leqq 3 \pi, \\
u=-\varphi(\theta-3 \pi) & \text { for } 3 \pi \leqq \theta \leqq 4 \pi,
\end{array}
$$

\section{§. Analytical expression of $\varphi(\theta)$.}

Finally the explicit analytical expression of the solution will be given.

By putting $\sin ^{2}(\theta / 2)=z,(3.1)$ is transformed into

$$
z(1-z) \frac{d^{2} w}{d z^{2}}+\frac{1}{2}(1-2 z) \frac{d w}{d z}-c\left[\frac{a}{4 z(1-z)}+b\right] w=0 .
$$

Again by putting $w=z^{\lambda_{1} / 2}(1-z)^{\left(1-\lambda_{1}\right) / 2} \cdot W,(9.1)$ is reduced to

$$
z(1-z) \frac{d^{2} W}{d z^{2}}+\left(\lambda_{1}+\frac{1}{2}-2 z\right) \frac{d W}{d z}-\frac{1+4 b c}{4} W=0 .
$$

This is a well-known Gauss' hypergeometric differential equation.

Since $u=\varphi(\theta)$ which represents a curve $C_{1}$ is of the form

$$
u=\frac{c \theta}{\lambda_{1}}+\cdots
$$

corresponding $w$ can be expressed in a form

$$
w=\text { const } \times \theta^{\lambda_{1}}(1+\cdots)=\text { const } \times z^{\lambda_{1} / 2}(1+\cdots)
$$

in the vicinity of $\theta=0$. Thus, in turn, corresponding $W$ should be a solution of (9. 2) such that

$$
W=1+\cdots
$$

in the vicinity of $z=0$ where the terms not explicitly written are power series of $z$. Such a solution of (9.2) is obviously given by a hypergeometric function

$$
\begin{gathered}
W=F(\alpha, \beta, \gamma ; z), \\
\alpha=\frac{1}{2}+i \sqrt{b c}, \quad \beta=\frac{1}{2}-i \sqrt{b c}, \quad \gamma=\frac{2+\sqrt{1+4 a c}}{2} .
\end{gathered}
$$

Thus we immediately have

$$
u=\varphi(\theta)=c \sin \theta\left[\frac{1+\sqrt{1+4 a c}}{2}-\sin ^{2} \frac{\theta}{2}\right.
$$




$$
\left.+2 \sin ^{2} \frac{\theta}{2} \cos ^{2} \frac{\theta}{2} \frac{F^{\prime}\left(\alpha, \beta, \gamma ; \sin ^{2}(\theta / 2)\right)}{F\left(\alpha, \beta, \gamma ; \sin ^{2}(\theta / 2)\right)}\right]^{-1} \text {. }
$$

Acknowledgement. The author is indebted to Prof. Tosiya Saito of Tokyo Metropolitan University for the proper guidance.

\section{REFERENCE}

[1] IkeBe, Y., On some fundamental properties of valve-controlled integral-type hydraulic servomotors. Bull. T.I.T. (B) (1956), 93-98.

National Defense College, Tokyo. 